\title{
L'ouvrier en personne, une irruption dans le cinéma documentaire (1961-1974)
}

\author{
par Nicolas Hatzfeld, Gwenaële Rot et Alain Michel*
}

$\mathrm{E}$ n 1967, les dirigeants de Citroën demandent à la cinéaste Annie Tresgot $^{1}$ de produire un documentaire sur la situation des ouvriers-paysans de l'usine de Rennes, récemment plantée au cœur de la Bretagne rurale. La cinéaste fait appel à son confrère québécois Michel Brault. Leur coopération donne naissance au film Les enfants de Néant, qui raconte l'embauche d'un paysan pauvre et son entrée dans le monde industriel. Cinq ans plus tard, de sa propre initiative, le cinéaste Louis Malle obtient l'autorisation pour, à son tour, poser sa caméra dans la même usine. Il réalise Humain, trop humain, autre documentaire montrant des ouvrières et ouvriers aux prises avec l'organisation du travail. Ainsi, à quelques années d'écart, situées de part et d'autre de 1968, deux grands auteurs de documentaires offrent à voir des visions fort différentes d'une réalité sociale similaire.

Chacun à sa façon, ces réalisateurs accordent une grande importance au documentaire. Ils participent à la transformation majeure de ce genre, que l'on peut rapprocher de l'évolution du film ethnographique dont Chronique d'un été de Jean Rouch et Edgar Morin a marqué un temps fort en 1961. Des changements techniques permettent d'alléger considérablement les opérations de filmage, de rapprocher la caméra des scènes filmées et, ce faisant, d'explorer de nouvelles façons de représenter le réel. Les financements institutionnels jusque-là de rigueur et, partant, les cahiers des charges qui encadraient les projets se trouvent dès lors remis en cause. Les points de vue des réalisateurs gagnent en autonomie et visent à se rapprocher des personnes filmées, plus encore à faire intervenir celles-ci directement dans les films. Cette démarche trouve dans les usines, en particulier les usines automobiles, des occasions d'autant plus marquantes qu'elles sont rares. Elles font surgir des individualités ouvrières dont l'inscription dans la société française s'effectue sur un mode original. Ce mouvement du cinéma s'inscrit, avec un rythme et un parcours qui lui sont propres, dans des modifications qui affectent la société. Dans les années 68 , l'image de l'usine change, de fait, beaucoup plus que la réalité de ce qui s'y vit ${ }^{2}$. La comparaison des deux films rend compte de ce déplacement. La mise en correspondance de leur réception et de leurs carrières fait ressortir combien, en quelques années, les références de la critique changent de façon encore plus radicale.

* N. Hatzfeld et A. Michel sont maîtres de conférences en histoire contemporaine à l'Université d'Évry-Val d'Essonne et chercheurs au LHEST (Laboratoire d'Histoire Économique, Sociale et des Techniques). G. Rot est maîtresse de conférences en sociologie à l'Université Paris-Ouest Nanterre-La Défense et chercheuse à l' UMR IDHE (Institutions et dynamiques historiques de l'économie).

1. Productrice du film, Annie Tresgot négocie celui-ci avec Citroën, et en assure également le montage. Annie Tresgot, entretien avec les auteurs, juin 2008.

2. N. HatzFELD, «Les ouvriers de l'automobile : des vitrines sociales à la condition des OS, le changement des regards ", in G. Dreyfus-Armand, R. Frank, M.-F. LÉvy, M. Zancarini-Fournel (dir.), Les années 68. Le temps de la contestation, Bruxelles, Éditions Complexe - IHTP CNRS, 2000, p. 345-362. 


\section{Une usine, deux représentations}

Se réclamant d'un même genre de réalisme, les deux films se distinguent par leurs procédés narratifs.

Les Enfants de Néant est tourné en 1967 et au début de 1968, en deux temps et dans deux lieux, le village de Néant-sur-Yvel dans le Morbihan et les ateliers de l'usine Citroën à Rennes, qui est alors l'usine automobile la plus moderne de France $^{3}$. Le film évoque la grande mutation du monde paysan confronté à de graves difficultés économiques à travers le cas de Joseph Leborgne. Ce dernier, comme de nombreux paysans bretons ne pouvant plus vivre de leur activité agricole, se résout à se faire embaucher à l'usine qui emploie à l'époque plus de six mille personnes. Brault scrute les étapes de sa mutation en ouvrier à travers cinq séquences tournées dans l'usine : l'embauche, la présentation des horaires et du travail par la hiérarchie, la visite des ateliers, l'apprentissage maladroit du poste et, six mois plus tard, Joseph, maîtrisant avec dextérité son pistolet de peinture. À partir des images filmées par le cinéaste, la productrice Annie Tresgot, elle-même réalisatrice d'autres films, se charge du montage et construit un documentaire social atypique, jouant sur l'alternance. Tout au long du film, les séquences d'usine sont intercalées avec celles qui présentent le monde rural que ce paysan-ouvrier ne quitte pas vraiment : son village, sa maison, ses champs qu'il continue à cultiver le week-end avec son frère. Le film emprunte à la fois aux genres du portrait et du récit, ajoutant aux scènes prises sur le vif des entretiens filmés ainsi que le réemploi de films et de photographies ${ }^{4} \mathrm{~d}^{\text {'archives sur }}$ les luttes paysannes, commentées en voix off. Le contraste entre les mondes des champs et de l'usine est accentué par les choix musicaux : des chants accompagnés à la harpe celtique d'un côté, de l'autre des pièces originales du compositeur Luc Perini suggérant les bruits d'usine.

Louis Malle, qui a visionné ce film avant d'entreprendre le sien ${ }^{5}$, souhaite privilégier dans Humain, trop humain une approche plurielle du travail, centrée sur l'activité proprement dite : "Nous voulions montrer les gens en train de travailler et faire sentir le rythme du travail à la chaîne, ce que ne faisait pas un film par ailleurs très réussi sur le plan de l'enquête sociologique comme Les Enfants de Néant " ${ }^{6}$. De fait, posant sa caméra dans les ateliers, Louis Malle s'attache à restituer les gestes, les visages et les regards d'hommes et de femmes qui travaillent à la chaîne, sur fond de bruits d'usine et sans le moindre commentaire. Le film est en effet divisé en trois sections distinctes ${ }^{7}$. Dans la première partie, Malle filme une succession d'ateliers selon un ordre correspondant à une logique convenue de fabrication, du stockage des tôles jusqu'à l'assemblage final, à l'instar de nombreux films d'entreprise ${ }^{8}$. Les principales opérations de fabrication sont montrées à partir des tâches et des gestes

3. Cf. P. Caro, Les usines Citroën de Rennes : origines, implantations et évolutions (1951-1974), mémoire de DEA d'histoire (dir. P. Fridenson), École des Hautes Études en Sciences Sociales, 1993.

4. D’Élie Kagan, cité au générique.

5. Archives Louis Malle 0289 B 78, Bibliothèque du film, Cinémathèque française, Paris.

6. L. Malle, interview «En direct avec Louis Malle », Écran, 25 mai 1974, p. 26.

7. Pour une présentation plus précise du film, cf. N. Hatzfeld, G. Rot et A. Michel, « Le travail au premier plan. Humain, trop humain de Louis Malle, 1972 ", Le Temps des Médias, n 7, hiver 2006-2007, p. 218-227.

8. N. Hatzfeld, G. Rot et A. Michel, «Filmer le travail au nom de l'entreprise ? Les films Renault sur les chaînes de production ", Entreprises et Histoire, n ${ }^{\circ 4}$, septembre 2006, p. 25-42. 
des ouvriers, sans le moindre mot de ceux-ci ni d'aucune voix off. La seconde partie du film se transporte au Salon de l'Automobile, où elle montre et fait entendre les conversations des visiteurs du stand Citroën. Dans la troisième partie, Malle retourne à l'usine, de nouveau sans la moindre parole, en réalisant différents portraits d'ouvrières et d'ouvriers en activité. En s'attachant de près à leurs visages et à leurs gestes, la caméra concentre son propos sur l'engagement des corps au travail. Ce troisième temps déplace ainsi le point de vue sur l'usine, considérée ici à travers des personnes placées à l'avant-scène. Louis Malle se refuse, dans l'ensemble, à la variété de procédés mis en œuvre dans Les Enfants de Néant, renonçant même à quelques entretiens effectués pendant le tournage ${ }^{9}$ afin de privilégier " un sentiment physique de fatigue et d'ennui - cet aspect totalement inhumain d'une chaîne de montage - sans avoir à l'exprimer par des mots, mais seulement en le montrant d'une manière très directe par l'image et le son ${ }^{10}$.

Les deux représentations de l'usine different radicalement. Ne s'embarrassant pas d'une mise en contexte, Louis Malle donne à la sienne une dimension universelle et relativement intemporelle. Avec Brault on suit à travers un personnage la double vie de paysans happés par l'industrialisation des Trente glorieuses. Dans cette manière de filmer les hommes au plus près de ce qu'ils expriment, les cinéastes ont en partage certains procédés et modes d'approche du cinéma direct.

\section{En partage, une filiation du film documentaire}

Louis Malle commence son film par un hommage explicite aux Enfants de Néant. Dans l'ouverture de Humain, trop humain, une pontonnière aux airs de madone assure l'approvisionnement des ateliers en rouleaux de tôle : le visage empreint d'une attention paisible, elle veille sur le hall de stockage et déplace l'appareil sur lequel elle se tient debout en actionnant les manettes par des gestes précis. Cette scène reprend une séquence tournée en noir et blanc par Brault. Renforcée chez celui-ci par des chants bretons introduits avec des images de calvaires livrés au vent, l'évocation religieuse est reprise chez Malle, cette fois à propos de l'usine elle-même, par des chants grégoriens qui accompagnent l'image de la même femme présente au même poste, comme immuable, cinq ans après. Derrière cette citation se trouve l'attachement partagé par les deux réalisateurs pour le cinéma direct.

C'est en 1955, à seulement 23 ans, que Louis Malle réalise avec le commandant Cousteau Le monde du silence, son premier film. Les équipements innovants permettent l'exploration inédite des fonds sous-marins qui fait la célébrité du film primé à Cannes. Pour un temps, c'est cependant vers la fiction que se tourne Malle. Peu après, Michel Brault, qui travaille à l'Office national du film du Canada (ONF), ponctue ses débuts de réalisateur en supprimant le trépied de sa caméra, pourtant une lourde $35 \mathrm{~mm}$, pour filmer Les Raquetteurs (1958). Se faufilant entre les gens, il s'efforce de faire vivre de l'intérieur les moments qu'il filme. Parallèlement, le preneur de son Marcel Carrière réussit à enregistrer dans certaines séquences un son synchronisé avec l'image. Les procédés, novateurs là aussi, marquent le monde du

9. Il s'agit du directeur de l'usine et d'un ouvrier-peintre. Entretien de J.-C. Laureux avec les auteurs, janvier 2004. Cf. N. Hatzfeld, G. Rot, A. Michel, «Entretien avec Jean-Claude Laureux preneur de sons ", La revue documentaire, $\mathrm{n}^{\circ}$ 21, 2007, p. 134-143.

10. P. French, Conversations avec... Louis Malle, Paris, Denoël, 1993, p. 190. 
cinéma. Brault ne tarde pas à croiser la route d'un autre pionnier du cinéma direct ${ }^{11}$, Mario Ruspoli, pour lequel il prend les images de deux autres documentaires : Les inconnus de la terre, qui évoque la condition et la culture de paysans en Lozère, et Regard sur la folie. Ces deux films sortis en 1961 sont remarqués, au-delà du monde du cinéma, dans les milieux intellectuels. Mais entre-temps a lieu le tournage de Chronique d'un été de Jean Rouch et Edgar Morin, qui joue un rôle décisif pour la formation du cinéma direct.

Avant de travailler avec Jean Rouch, Michel Brault connaissait et appréciait ses films, tandis que celui-ci voit Les Raquetteurs au séminaire sur Flaherty en Californie durant l'été 1959. "Lorsque j'ai vu les films de Rouch, j'en ai eu un peu le coup de foudre, mais ça n'a pas été suffisant pour qu'on se rencontre. À partir du moment où Rouch a vu Les Raquetteurs, on a commencé à se comprendre, parce qu'on s'est aperçu qu'on se ressemblait un peu comme cinéastes : la même attitude fondamentale de filmer la vie telle qu'elle est. [...] Jean Rouch cherchait quelqu'un pour tenir sa caméra; il cherchait un gars qui aurait un peu le même œil que lui " ${ }^{12}$. Ailleurs, Michel Brault précise les raisons du choix de Rouch : "En voyant Les Raquetteurs, il a constaté que je travaillais au grand angulaire. Pour travailler au grand angulaire, il faut que tu voies les gens, que tu sois près d'eux. Si tu arrives à les filmer et qu'ils continuent à vivre pendant que tu es près d'eux, c'est qu'ils t'ont accepté dans le groupe " ${ }^{13}$.

Cette reconnaissance réciproque renvoie à l'engagement voisin de ces hommes de cinéma dans le mouvement de transformation du film documentaire. Depuis $L a$ sortie de l'usine Lumière à Lyon, filmée en 1895, l'idée de filmer la vie telle qu'elle est accompagne les projets, les entreprises et les courants du cinéma ${ }^{14}$. Les jeunes cinéastes des années 1950 se reconnaissent héritiers de Robert Flaherty ou de Dziga Vertov, pionniers dans les années 1920 d'un cinéma fondé sur la rencontre, dont les deux options (saisir « la vie au naturel » pour le premier, et pour le second « la vie à l'improviste ${ }^{15}$ ) irriguent les types de pratique ou les références théoriques ${ }^{16}$. Les diverses variantes de l'engagement, du militantisme au réalisme poétique, nourrissent les échanges dans ce genre qualifié pour la première fois de documentaire en 1926 par le britannique John Grierson, ou peut-être plus tôt par Louis Delluc en $1923^{17}$. Quel que soit son premier auteur, le mot portait sur le film Nanouk de Flaherty. Depuis, il désigne la représentation, au besoin dramatisée, de la vie de

11. E. Michaud indique que «le terme cinéma en prise directe, suggéré en 1963 par Mario Ruspoli, provient d'une analogie d'ordre mécanique : la prise directe désigne la quatrième vitesse en voiture. D'où un cinéma en prise directe sur le réel, capté sur le vif ". Cf. E. Michaud, "Les débuts du direct ", Cabiers du Gerse, Du simple au double, n 1, 1995.

12. "Michel Brault et Claude Jutra racontent Jean Rouch ", Objectif, n³, 1960, p. 4.

13. M. Brault, "Entretien avec Arthur Lamothe ", La Presse, 18 août 1961. Cité dans F. Niney, L'épreuve du réel à l'écran, Bruxelles, De Boeck, 2002, p. 139. Ce choix est le résultat d'une remise en question des pratiques filmiques : «On était des gamins, on faisait des films pour la télévision et on riait des gens, on filmait dans la rue leurs travers au téléobjectif. On volait des images. Jusqu'au jour où on s'est dit : "Non. Qu'est-ce que c'est, ce vol d'intimité ?". Alors là, la seule solution c'est de changer le téléobjectif pour le grand angulaire ». Michel Brault, entretien avec G. Rot et M. Julien, juin 2008.

14. Avant les frères Lumière, Jules-Étienne Marey et Georges Demeny saisissent des mouvements mécaniques, animaux et surtout humains. Mais, pensant en scientifique, Marey refuse la simple représentation cinématographique du réel destinée à alimenter une activité de spectacle.

15. F. Niney, L'épreuve, op. cit., p. 47-50.

16. G. Gauthier, Un siècle de documentaire français, Paris, Armand Colin, 2004, p. 149.

17. L. Delluc, Écrits cinématographiques II : Cinéma et Cie. Le cinéma au quotidien, Paris, Éditions de la Cinémathèque - Éditions de l'Étoile Cahiers du cinéma, 1986. 
personnes réelles. Les débats et les expériences sont toutefois en train de changer à partir de la fin des années 1950.

\section{Cinéma direct et ethnographie, de nouvelles combinaisons}

Depuis l'après-guerre, certains techniciens et réalisateurs s'activent à transformer les conditions de filmage, à travers le développement d'innovations convergentes. La sensibilité des pellicules fait l'objet de demandes et d'expérimentations, en vue d'affranchir les tournages des contraintes de lourds éclairages en studio. Parallèlement, les caméras font l'objet d'attentes et de recherches faisant coopérer fabricants, opérateurs et réalisateurs. Il s'agit d'alléger le maniement des caméras, dont l'énorme poids empêchait l'utilisation à bout de bras. À la suite d'innovations de guerre (caméras embarquées à bord d'avions de reconnaissance ou de bombardement), arrivent des caméras plus légères, telle celle qu'utilise Jean-Luc Godard dans $A$ bout de souffle ${ }^{18}$. L'allègement s'obtient d'abord au prix d'un bruit accru, qui rend à peu près impossible toute prise de son directe.

Dans ce mouvement, la dynamique ethnologique se combine avec celle des cinéastes. Dès le temps de la Seconde Guerre mondiale André Leroi-Gourhan a relevé l'intérêt que les ethnologues pouvaient avoir à utiliser des caméras $16 \mathrm{~mm}$ dans leurs enquêtes de terrain ${ }^{19}$. Jean Rouch concrétise avec d'autres cette propension ethnologique au cinéma, par ses propres réalisations et par la multiplication d'initiatives internationales en faveur d'un développement du film ethnographique ${ }^{20}$. Parallèlement, il s'emploie à transformer l'outillage disponible afin d'enrichir les possibilités de ce genre. Selon toute vraisemblance, c'est une des raisons pour lui de faire appel à Michel Brault, qu'il espère aussi mettre à contribution pour le développement d'une caméra permettant une prise de son synchrone ${ }^{21}$. Il coopère déjà avec André Coutant, ingénieur chez le fabricant Éclair, afin de gagner en légèreté, en maniabilité et en silence. Parallèlement, il est - apparemment comme Brault ${ }^{22}$ - en relation avec un autre ingénieur, Stefan Kudelski, qui vient de mettre au point sa troisième génération du magnétophone Nagra, entièrement transistorisé et fonctionnant sur pile, d'une qualité égale à celle des enregistreurs de studio. Pour la première fois, le rêve d'une prise de son effectuée directement dans des situations réelles est à portée de main. C'est à cette possibilité d'en finir avec les sons et dialogues reconstitués en studio que renvoie la formule de cinéma direct. Là se joue l'effet de réalité des films documentaires.

À l'occasion du tournage de Chronique d'un été, Michel Brault et Jean Rouch ${ }^{23}$ sont amenés à collaborer pour perfectionner les outils et les techniques. "Michel Brault nous apporta un micro utilisé par la TV canadienne appelé micro cravate.

18. A. Bergala, Godard au travail, Paris, Éditions des Cahiers du Cinéma, 2006.

19. Ce point est rappelé par G. GAuthiER et al., Le documentaire passe au direct, Montréal, VLB éditeur, 2003, p. 59.

20. L. DE Heusch, «Jean Rouch et la naissance de l'anthropologie visuelle. Brève histoire du Comité international du film ethnographique ", L'Homme, nº 180, octobre-décembre 2006, p. 43-71.

21. R.Q., "Avec Chronique d'un été, Jean Rouch fait une tentative sociologique », Combat, 11 novembre 1960, p. 2. Cité dans A. Loiselle, Le cinéma de Michel Brault, à l'image d’une nation, Paris, L'Harmattan, 2005, p. 62.

22. Annie Tresgot, entretien cité.

23. Sur les liens entre Rouch et les cinéastes de l'ONF, cf. V. Bouchard, « Jean Rouch et l'Office National du Film ", Hors Champ, novembre 2004. 
[...] Il nous a aussi apporté une technique qu'il avait mise au point au Canada depuis longtemps, celle de la "caméra qui marche". Il s'était entrâné pendant un an à marcher en avant, en arrière de côté, si bien qu'entre ses mains la caméra devenait absolument mobile tout en restant parfaitement stable " ${ }^{24}$. La caméra, dont le bruit de fonctionnement gêne la prise de son, fait alors l'objet des attentions combinées des cinéastes et de l'ingénieur André Coutant : "Nous avons commencé à tourner avec cette caméra. On tournait. On discutait. C'était vraiment extraordinaire. [...] Nous allions le soir à l'atelier de Monsieur Coutant et nous lui demandions s'il ne pouvait pas ajouter ceci ou modifier cela. Monsieur Coutant se prêtait aimablement à tous nos désirs " ${ }^{25}$. De fait, de perfectionnement en audace expérimentale, le film est l'occasion de concrétiser pour la première fois, sur certaines séquences, les traits du nouveau cinéma documentaire, que la mise au point de caméras $16 \mathrm{~mm}$ facilite.

La dynamique technique ne passionne Rouch et Brault que pour autant qu'elle sert un projet cinématographique. À cet égard, Chronique d'un été, auquel contribue un alliage de personnalités, marque tant les sciences sociales que les milieux du documentaire $^{26}$. On se contentera d'évoquer la définition qu'en donne Edgar Morin : "Ce n'est pas un film documentaire. Cette recherche ne vise pas à décrire; c'est une expérience vécue par ses auteurs et ses acteurs. Ce n'est pas un film sociologique à proprement parler. Le film sociologique recherche la société. C'est un film ethnologique au sens fort : il cherche l'homme. C'est une expérience d'interrogation cinématographique : comment vis-tu ? ${ }^{27}$. Primé dans plusieurs festivals, le film fait référence à bien des égards. Il conforte le projet de cinéma direct s'appuyant sur le couplage sonimage et fait passer le documentaire, pour reprendre la formule de François Niney, du parlé au parlant ${ }^{28}$. Ce faisant, il estompe la frontière entre filmeurs et filmés, les réalisateurs entrant dans certaines scènes tandis que les interviewés se font à maintes occasions interviewers. Le genre documentaire s'en trouve bouleversé.

\section{Des rencontres renouvelées avec l'usine}

Dans Chronique d'un été, Angelo, qui travaille à Renault-Billancourt, fait le pendant ouvrier aux autres personnages. Et, illico, met à mal la figure prolétaire, stigmatisant pour l'étudiant africain auquel il s'adresse le travail et l'ambiance de l'usine : « Je me demande comment on peut faire pour arriver à rester, dans une usine. Comme vous dites, enfermé. On est contrôlé, et il y a cette espèce de division qui se crée déjà, entre ouvriers. En plus de ça, il y a la maîtrise qui nous harcèle, qui est toujours derrière nous, les contremaîtres ". Angelo continue, en insistant sur ses collègues de travail : «Il faut compter... $80 \%$ de types qui ont leur voiture. Parce qu'il faut se dire qu'ici, en France, le type est individuel. Il travaille pour lui, il ne pense qu'à lui. Il a son salaire, il travaille. Il bosse, il amasse du pognon, il se prive sur certaines

24. "Jean Rouch, le bon génie des personnages en liberté surveillée ". Interview effectué par Jean Grob assisté de Jean Mazéas, Le cinéma pratique chez soi, n 39, 1962, p. 53-54.

25. M. Brault, cité dans G. Marsolais, Michel Brault, Québec, Conseil québécois pour la diffusion du cinéma, 1972, p. 26.

26. W. Rothman, Documentary film classics, Cambridge, Cambridge University Press, 1997, p. 69-108.

27. E. Morin, "Chronique d'un film », in E. Morin et J. Rouch, Chronique d'un été, Paris, Interspectacles, 1962, p. 8-9.

28. F. Niney, L'épreuve du réel, op. cit., p. 69-168. 
choses. Il veut jouer à cette espèce de type qui a du pognon, qui a du fric. Tu vois, c'est un pauvre type ». Le rejet de sa propre identité sociale, la représentation fragmentée et individualiste des relations de travail contredisent la dimension collective et organisée de la figure ouvrière en lutte.

Cette parole désenchantée porte d'autant plus qu'elle fait irruption à une période où la " silhouette ouvrière " est absente, ou presque, de la production cinématographique française de fiction, entre les deux temps plus fournis des années de Reconstruction et des années $68^{29}$. Ailleurs cependant, dans le genre documentaire, les vues d'atelier ne manquent pas. Elles proviennent en majeure partie de films commandités par les entreprises ou même, comme chez Renault, directement réalisés par un service propre, et qui se trouvent ensuite distribués, sous le label documentaire, dans les salles de cinéma en avant-programme ${ }^{30}$. Ces courts métrages à double fond offrent, fort logiquement, une image positive des ateliers. Mais, pris dans la grandiloquence, ils négligent généralement les ouvriers, dissous dans la démonstration de ce qui est au cœur des discours, l'efficacité industrielle ${ }^{31}$. À travers l'intervention personnalisée de l'ouvrier Angelo, Chronique d'un été fait ressortir les biais de la production antérieure, encombrée par les lourdeurs techniques, les coûts de réalisation, le poids du financement institutionnel et les accommodements dans le choix et le traitement des sujets. Reléguées dans la catégorie des films d'entreprise, ces manières des années 1950 ouvrent la voie à une redécouverte.

Aussi, lorsque le PDG de Citroën, Pierre Bercot, sollicite Annie Tresgot pour un film ayant le double statut de film d'entreprise et de documentaire, cherche-t-elle à éviter ce qu'elle voit comme un double piège : le risque de réaliser un film désormais difficilement recevable dans le monde du documentaire et, avant cela, le risque de ne pouvoir surmonter les crispations de l'encadrement local. Celui-ci est marqué par un syndicalisme corporatif suivant le modèle répressif ${ }^{32}$ en vigueur chez Simca. " Je n’ai pas voulu le réaliser, parce que j'ai pensé que c'était piégé, à cause des résistances du milieu. J'ai pensé à Michel Brault, qui était un peu le pape des nouvelles technologies, et d'une nouvelle approche. [...] Par ailleurs, Brault avait l'avantage de ne pas être français, et donc de ne pas être impliqué dans ces luttes syndicales que le patronat redoute tant $"{ }^{33}$. L'option choisie de suivre la formation d'un ouvrier-paysan satisfait le patron désireux de montrer le rôle de l'entreprise pour la dynamisation industrielle de la Bretagne empêtrée dans sa ruralité. "La population de Rennes fournit peu d'effectif. C'est toute la région qui envoya ses hommes. De petits villages fournirent des contingents importants, ces hommes travaillant 8 heures à l'usine et complétant souvent dans le reste de la journée, ou le samedi, le travail que l'épouse fournissait pour une petite exploitation qui souvent ne dépassait pas cinq hectares. [...] L'équilibre entre une petite exploitation familiale et le salaire complémentaire

29. M. CADÉ, L'écran bleu. La représentation des ouvriers dans le cinéma français, Perpignan, Presses Universitaires de Perpignan, 2004, p. 56.

30. N. Hatzfeld, G. Rot, A. Michel, "Filmer le travail au nom de l'entreprise... », art. cit.

31. Dans un film de commande réalisé pour Pechiney, Le chant du styrène (1958), A. Resnais parvient néanmoins à ne pas estomper totalement la figure ouvrière. G. RoT, "Le travail de contrôleur de flux, de Pierre Naville à Alain Resnais. Propos sur Le Chant du styrène ", Histoire et société. Revue européenne d'histoire sociale, $\mathrm{n}^{\circ}$ 24, 2007, p. 52-64.

32. J. Coutouly, Les paysans-ouvriers et l'agriculture, Informations agricoles et économiques, document multigraphié, 12 août 1966, 11 p. Archives personnelles d'A. Tresgot.

33. Annie Tresgot, entretien cité. 
d'un homme qui n'avait pas besoin de connaitre un métier fit de la région un pays qui devint sans problèmes, et de l'usine de La Janais une usine sans grèves " ${ }^{34}$. Forte de ce soutien, l'équipe résiste aux pressions de l'encadrement qui cherche à l'expédier " filmer les binious " : tout en filmant avec intérêt les festivités et sites traditionnels, Brault continue de promener sa caméra dans les ateliers. Loption cinématographique alterne deux principaux usages. D'une part une grosse caméra $35 \mathrm{~mm}$, souvent munie d'un téléobjectif permettant de saisir en gros plan des gestes et des visages d'une haute qualité visuelle, et d'autre part une $16 \mathrm{~mm}$ avec son synchrone pour les séquences de suivi rapproché. Elle permet de faire jouer les idéaux-types chers au style de Flaherty, la campagne du fond des âges et la modernité industrielle, combinées dans la figure de l'ouvrier-paysan. L'approche du cinéma direct apporte l'expression de l'attachement à la ferme, du désarroi dans l'atelier et, somme toute, d'un accommodement fataliste : «Finalement, l'image parle plus que les mots. Joseph n'avait pas une conversation très abondante. N'empêche qu'il était, derrière cela, un être humain qui subissait. [...] Il est un peu, comment dire, résolu : il faut s'y faire $"^{35}$. Enfin, délibérément ou non, le film soulève des questions d'histoire du travail. Montrant le héros peindre en cabine, le visage nu malgré la toxicité des produits, des pièces métalliques défilant à la chaîne, il pourrait inciter à supposer une prise de risque en milieu ouvrier. Sur cette hypothèse, le patron remet les repères en place en vantant, dans ses ateliers de peinture, les cabines fonctionnant " avec des conditions d'aération qui permettaient de travailler sans masque ${ }^{36}$. Filmant le même secteur, Louis Malle fait ressortir grâce à la couleur le visage bleui d'un jeune peintre.

Louis Malle s'inscrit dans une relative continuité du cinéma direct, dont il adopte les évolutions dès qu'il reprend la réalisation de documentaires. L'équipe rapprochée qu'il constitue vient du réseau pionnier. Dès le tournage de Calcutta, il s'associe avec Étienne Becker « qui appartient à cette race de nouveaux opérateurs dont la démarche relève presque de la danse» ${ }^{37}$, ayant travaillé avec Jean Rouch ${ }^{38}$, Mario Ruspoli ${ }^{39}$, Chris Marker ${ }^{40}$ ou encore Joris Ivens. Jean-Claude Laureux, ingénieur du son comparse de Becker, a travaillé lui aussi avec Mario Ruspoli, et pratique le son direct, associant le Nagra avec la caméra Coutant que les mouvements de mai-juin 68 banalisent. Suzanne Baron a dès ses débuts travaillé avec Rouch, qu'elle a accompagné pendant deux ans au Musée de l'Homme, et devient la monteuse attitrée de Louis Malle. Ainsi la sensibilité aux mêmes façons de faire offre-t-elle d'emblée une souplesse d'action que revendique Malle : "Ce que j'appelle du cinéma direct est une forme de documentaire où l'on improvise totalement, où l'on travaille avec une équipe minimum, où l'on cherche non pas à organiser la réalité, mais simplement à aller là où vous entraîne votre curiosité. On essaie de filmer sur le vif ce qui paraît surprenant ou intéressant, et ensuite on tâche de donner un sens à tout ça, dans la salle de montage. C'est un cinéma de l'instinct, de l'improvisation, un cinéma

34. P. Bercot, Mes années aux usines Citroën, Paris, La pensée universelle, 1977, p. 60.

35. Michel Brault, entretien avec Gwenaële Rot et Michel Julien, mai 2008.

36. P. Bercot, Mes années..., op. cit., p. 58.

37. L. Marcorelles, Une esthétique du réel, le cinéma direct, Rapport UNESCO WS/0964.90CUA, Paris, 12 octobre 1964, p. 20.

38. Paris vu par, 1963 [sec 2 'Gare du Nord' par Jean Rouch] 16mm-3.

39. Segestre, 1965 , court métrage de $11 \mathrm{mn}$.

40. Autre signe des circulations : Étienne Becker joue dans La Jetée de Chris Marker (1962). 
véritablement du présent ${ }^{41}$. La connivence au sein du trio de tournage permet parfois à l'un de ses membres d'entrer en action spontanément, et d'entraîner les autres à sa suite ${ }^{42}$. Toutefois la rencontre avec les ouvrières et ouvriers ne cherche pas à obtenir une co-réalisation ${ }^{43}$. Effectuant un séjour ramassé d'une quinzaine de jours, l'équipe se contente de se faire admettre par ceux qu'elle filme, visant à ce que son intrusion perturbe le moins possible l'ordre des choses : «La difficulté, quand on vient filmer quelque part, c'est que c'est nous l'événement, justement. Ils sont dans leur quotidien, et tout à coup, des gens arrivent. [...] Donc les regards se tournent vers nous. On ne peut pas faire autrement, mais en tout cas, on essayait d'être le plus discret, le plus inexistant possible ${ }^{44}$. Plus encore que la caméra qui peut recourir au téléobjectif et rester à distance, la perche de l'ingénieur du son signale l'action des cinéastes et suscite la réserve des intéressés : "La première impression est globale. Une espèce d'énorme bruit. On n'arrive pas encore à faire tout de suite la différence entre les sons. [...] Je me suis demandé ce que j'allais pouvoir sortir, avec une telle masse sonore. En fait, il faut se rapprocher des choses. Quand on se rapproche vraiment d'un outil, il devient plus fort et à ce moment-là, c'est l'aspect musical qui prend le dessus» ${ }^{45}$. Euvrant en franc-tireur du documentaire, Malle veille à aménager l'espace de son intervention. C'est par son nom et sans doute des relations familiales qu'il obtient des dirigeants de l'entreprise l'autorisation de filmer, après avoir essuyé des refus tel celui de la Régie Renault. Il prend soin, apparemment, de contrebalancer cette introduction patronale par des contacts avec des syndicalistes de la CGT, grâce à l'entremise du cinéaste militant René Vautier ${ }^{46}$.

\section{Deux films dans la radicalisation des années 68}

Distribués respectivement en 1968 et 1974, les deux films suscitent des échos dont la différence tient beaucoup aux progrès de l'esprit contestataire issu de mai-juin. Le film de Michel Brault connaît un succès d'estime en France en tant que documentaire, tandis que l'entreprise renonce dans un premier temps à l'utiliser comme outil de communication : "C'est un beau film, que nous ne montrerons pas ", commence par dire le commanditaire, avant d'en demander malgré tout des copies ${ }^{47}$. L'œuvre est sélectionnée pour Cannes, puis reçoit un prix à la Quinzaine de la revue Positif. Si elle n'est pas distribuée au Québec, elle est diffusée, au nom de la France, par les télévisions du réseau francophone.

41. P. French, Conversation avec..., op. cit., p. 186.

42. Dès 1963, Mario Ruspoli théorise la nécessité d'une forte cohésion dans son rapport pour l'UNESCO. M. Ruspoli, Le groupe synchrone cinématographique léger, 1963. Cité par G. GaUTHIER, P. Pilard et S. Suchet, Le documentaire passe au direct, Paris, VLB éditeur, 1984, p. 63.

43. En ce qui concerne les façons d'accéder aux ateliers, de tourner, d'organiser le montage ainsi que sur l'accueil de la critique à la sortie du film, cf. N. Hatzfeld, G. Rot et A. Michel, "Le travail au premier plan..." ", art. cit.

44. Jean-Claude Laureux, entretien cité.

45. Ibid.

46. René Vautier, témoignage à l'occasion de la présentation de son film réalisé avec Soizig Chappedelaine Quand les femmes ont pris la colère (1977) au festival de cinéma Résonance à Bobigny en 2004. Ce film reprend de longues séquences d'Humain, trop humain.

47. Annie Tresgot, entretien cité. 
Les Enfants de Néant sont programmés au printemps 1968 au ciné-club Action en même temps qu'À bientôt jespère de Chris Marker qui rend compte, à travers des portraits d'ouvriers syndicalistes, de la grève de la Rhodiaceta en 1967. Le critique de Positif qui mentionne cette programmation concomitante souligne la parenté de ces deux films : "Il n'existe pratiquement pas de films parlant de la condition ouvrière en Petite Europe de nos jours ". L'auteur souligne ensuite que les deux œuvres recourent à la technique du cinéma direct, donnant la part belle à l'interview. Le statut de film de commande ne nuit pas à l'évaluation des Enfants de Néant, bien au contraire. Les critiques saluent le dépassement du genre et la liberté prise par le réalisateur vis-à-vis du commanditaire. Le journaliste des Cabiers du cinéma explique que le réalisateur, "mis en contact avec les dures réalités du pays, n’allait pas s'en tenir là " car ce qui est montré est le passage " de la misérable indépendance du petit paysan au moderne servage de l'ouvrier salarié ». Le film est apprécié non seulement pour sa maîtrise esthétique et son apport documentaire mais aussi parce qu'il joue un tour à ses commanditaires. Le critique des Cahiers relève "la technique de l'approche et de l'enveloppement doucereux " pratiquée par les cadres, tandis que celui de Cinéma 68 souligne également le caractère subversif du regard de Brault : «Il paraît que les commanditaires ne se sont pas déclarés ravis du résultat. On les comprendrait à moins : le regard totalement objectif jeté par Brault sur un phénomène social est un regard attentif, terriblement révélateur, même sans aucun commentaire en guise de conclusion".

Le mouvement de mai-juin 68 bouscule de telles références tant en ce qui concerne la représentation des ouvriers qu’à propos du rôle des cinéastes. De fait, le parti pris de Malle traduit ce changement, qui disqualifie la réalisation d'un documentaire sur les ouvriers à la demande de leur patron, ou la présentation du travail à la chaîne comme une perspective positive. Tout en s'inscrivant dans ces nouveaux repères, le film de Louis Malle est cependant controversé et somme toute fort peu diffusé. Il doit sa sortie en salle au fait même que Louis Malle est propriétaire de l'unique salle où il est projeté (La Pagode). Si certains critiques considèrent ses images dénuées de commentaires terriblement accusatrices et saluent l'absence de didactisme, d'autres, au contraire, considèrent inacceptable le fait de ne pas dénoncer le système par un discours explicite, et peut-être plus encore l'absence de parole ouvrière directe. On condamne désormais le "cinéma-direct-passif " sans détour dans les Cahiers $d u$ cinéma, un cinéma où les O.S. "n'interviennent jamais sur le propos du film, ni sur son déroulement ". Montrer une oppression et l'exploitation qui s'accomplissent sans révolte, ne serait-ce pas s'en accommoder ? L'époque est au surlignage politique, et ce silence est interprété, dans la critique militante, comme une forme de complaisance ${ }^{48}$. Pour expliquer ces réactions, on ne peut non plus exclure la suspicion politique qui pèse sur Malle en raison de son origine sociale, alors que Brault, cinéaste engagé pour la cause québécoise, est protégé de telles préventions.

En 1974, lorsque sort Humain, trop humain, l'exigence exacerbée de parti pris politique a commencé à céder du terrain au désenchantement. Les remises en question d'engagements militants et les changements de la conjoncture sociale rendent peu réceptif aux intentions de Louis Malle. Entre l'attente d'une dénonciation convenue et l'inaptitude à repenser alors la condition ouvrière, le film ne trouve pas

48. N. Hatzfeld, G. Rot et A. Michel, "Le travail au premier plan ... ", art. cit. 
son public. La mésaventure d'Humain, trop humain, particulière dans sa forme, n'est pas exceptionnelle. D'autres films contestataires ou militants tournés dans les années 1968 choisissent de représenter l'usine et les ouvriers en se référant au cinéma direct, ou sur la base d'une implication de vrais ouvriers composant et retravaillant leur propre rôle ${ }^{49}$. Les uns, tel Marin Karmitz dans Camarades, font jouer des fictions par de vrais ouvriers dans de vrais ateliers, d'autres font composer par de vrais ouvriers des scénarios qu'ils interprètent eux-mêmes : c'est le cas du groupe Medvedkine. Au cours même de 68 des tournages de luttes et d'occupations ont été effectués en vue d'un film collectif, sous les auspices des États Généraux du cinéma ${ }^{50}$. Ils donnent la matière de films dont le montage - et le scénario qui le sous-tend - sont ensuite assumés par des individualités : ainsi Oser lutter, oser vaincre de Jean-Pierre Thorn ou 33 jours en mai de François Chardeaux. Dans tous les cas, les œuvres achevées ne touchent ensuite que des publics engagés voire militants, et leurs options suscitent chaque fois, pour le moins, des controverses politiques vives (Jean-Luc Godard, Un film comme les autres, octobre 1968 ; Paul Seban, La CGT en Mai, mai 1969) ${ }^{51}$. À travers elles, le postulat politique qui étend son emprise sur le genre documentaire s'exacerbe au point de fragmenter les débats et les auditoires. Paradoxalement, les années 68 qui relancent les rôles ouvriers sur la scène sociale et politique ${ }^{52}$ ne correspondent pas dans le domaine cinématographique à l'émergence de figures fédératrices.

Au cours des années 1960, considérées comme l'apogée de l'ouvriérisation de la société française ${ }^{53}$, les représentations cinématographiques des ouvriers connaissent des ruptures significatives. Le changement ne vient pas des œuvres de fiction qui négligent généralement ce thème, et plus encore à cette époque qu'aux temps de la Reconstruction ou, un peu plus tard, des années 68. Le film documentaire, lui, est affecté d'une mutation que le critique Louis Marcorelles qualifie alors d'étape décisive dans l'évolution du cinéma moderne. Deux éléments distincts s'y combinent : "d'une part une technique à base de caméra légère et de son synchrone, d'autre part une réflexion essentiellement basée, aussi bien au niveau de la prise de vue que du montage, sur le son, donc la parole inséparable de tout un milieu ambiant et d'une action physiquement vécue $" ~ " 54$. Cette combinaison suscite la mise en cause d'une tradition du documentaire, encombrée de matériels lourds et de coûts élevés, engoncée dans ses liens avec les financements institutionnels ou les grandes entreprises du cinéma. Les films portant sur les usines, en l'occurrence, exprimaient un lyrisme industriel dans lequel les ouvriers faisaient généralement office de décor ou de figurants : de la maind'œuvre en activité. Un tel discours s'accordait avec les stratégies de communication des entreprises.

49. N. Hatzfeld, G. Rot et A. Michel, "Le travail en représentation dans les films militants ", Histoire et sociétés, $\mathrm{n}^{\circ}$ 9, janvier 2004, p. 118-131.

50. S. LayerLe, "En l'autre bord. Filmer les forces d' opposition à Mai 68 ", Le Mouvement Social, avril-juin 2008, p. 11-16 et Caméras en lutte en mai 68: "par ailleurs, le cinéma est une arme ", Paris, Nouveau monde éditions, 2008.

51. S. LaYerle, Caméras en lutte, op. cit., p. 180-204.

52. X. VIGna, Linsubordination ouvrière. Essai d'histoire politique des usines dans les années 68 , Rennes, Presses Universitaires de Rennes, 2007.

53. G. NoIRIEL, "Préface, vingt ans après ", Les ouvriers dans la société française, XIX -XXe siècle, Paris, Le Seuil, $2^{\mathrm{e}}$ édition, 2002, p. IX.

54. L. Marcorelles, Une esthétique..., op. cit., p. 7. 
En filmant de plain-pied des scènes de la vie ordinaire, ce cinéma fait surgir des personnes à la fois banales et singulières, dont la parole en direct provoque un puissant effet de vérité. Il souligne combien le film d'avant sent l'artifice. Pourtant le terme de cinéma-vérité ne tente guère les partisans du nouveau cinéma : trop moral pour les uns, illusoire pour les autres. Chacun travaille à sa façon la rigueur d'une option dans la chaîne de relation allant du filmé au spectateur, et fait ainsi de sa composition une affaire personnelle. Dès avant 68 sur les écrans, les Trente glorieuses perdent de leur lustre à l'aune du quotidien ouvrier lorsque, trop rarement, tel ou tel cinéaste le filme à hauteur d'homme. C'est le cas pour Chronique d'un été, ou pour Les enfants de Néant. 68 démultiplie le mouvement en même temps qu'il le crispe. Toujours minoritaires, mais nettement plus nombreux sont les filmeurs tentés par l'usine. L'engagement documentaire attire vers les ateliers parmi les plus grands noms, hisse à la réalisation des techniciens d'avant, propulse à la caméra de simples étudiants. Les usines de Citroën, de Peugeot, de Renault ou de Simca s'incarnent désormais à travers des voix, des personnages, au sens théâtral du terme. Souvent, la tentation est trop forte d'en faire des figures politiques, d'instrumentaliser la parole, et les films d'usine des années 68 offrent un riche panorama des projets prolétaires. Dans cet ensemble, Humain, trop humain s'inscrit en décalage par rapport à cette époque de contestation où le cinéma direct s'oriente davantage vers un activisme en direction de la classe ouvrière. Installant le silence d'ouvriers dont les yeux, parfois, interpellent, il invite le spectateur à rechercher sa place, à retravailler le lien.

\section{Jacques JULLIARD \\ L'Argent, Dieu et le Diable : Face au monde moderne avec Péguy, Bernanos, Claudel}

Flammarion

ᄂ - - - - - - - - - - - - - - - - - - - - - - - - - J

Jacques GIRAULT

Bordeaux et la Commune

1870-1871

Mouvement ouvrier et idéologie républicaine au moment de la Commune de Paris

Avant-propos de Jacques Rougerie

Fanlac 